\title{
The Significance of Preoperative Serum Sodium and Hemoglobin in Outcomes of Upper Tract Urothelial Carcinoma: Multi-Center Analysis Between China and the United States
}

This article was published in the following Dove Press journal:

Cancer Management and Research

\author{
Dong Fang, $\mathbb{D}^{1, *}$ \\ Nirmish Singla, $2, *$ \\ Zhengqing Bao, (D) 1,3,* \\ Syed M Jafri, ${ }^{4}$ Xiaohong Su, \\ Zhenpeng Cao,' \\ Gengyan Xiong, (D) Lei Zhang,' \\ Solomon Woldu, (iD) ${ }^{2}$ \\ Ryan Hutchinson, ${ }^{2}$ \\ Arthur Sagalowsky, ${ }^{2}$ \\ Yair Lotan, ${ }^{2}$ Xuesong Li, \\ Jay D Raman, ${ }^{4}$ Vitaly Margulis, ${ }^{2}$ \\ Liqun Zhou ID \\ 'Department of Urology, Peking \\ University First Hospital, Institute of \\ Urology, Peking University, National \\ Urological Cancer Center, Beijing, \\ People's Republic of China; ${ }^{2}$ Department \\ of Urology, University of Texas \\ Southwestern Medical Center, Dallas, \\ TX, USA; ${ }^{3}$ Department of Urology, \\ Beijing Jishuitan Hospital, The Fourth \\ Medical College of Peking University, \\ Beijing, People's Republic of China; \\ ${ }^{4}$ Division of Urology, Penn State Milton \\ S. Hershey Medical Center, Hershey, PA, \\ USA
}

*These authors contributed equally to this work

Correspondence: Liqun Zhou

Department of Urology, Peking University First Hospital, Institute of Urology, Peking University, National Urological Cancer

Center, No. 8 Xishiku Street, Xicheng

District, Beijing 100034, People's Republic of China

Tel +86-10-83575006

Fax +86-10-6655I I26

Email zhoulqmail@sina.com
Purpose: To analyze the effect of preoperative serum sodium and hemoglobin on oncologic outcomes in upper tract urothelial carcinoma (UTUC) based on a multi-center cohort from China and the United States (U.S.).

Methods: We retrospectively reviewed the records of 775 patients with UTUC treated surgically at tertiary care medical facilities in China or the US from 1998 to 2015 . We analyzed associations of preoperative serum sodium and hemoglobin with clinicopathological characteristics, overall survival (OS), cancer-specific survival (CSS) and intravesical recurrence free survival (IVRFS).

Results: The US patients had comparatively lower serum sodium and similar hemoglobin at baseline. Preoperative low serum sodium value was associated with tumor multifocality, lymph node metastasis (LNM) and lymphovascular invasion (LVI); preoperative anemia was associated with advanced age, tumor multifocality, high tumor grade and LVI. Preoperative low serum sodium was an independent predictor of worse OS in the entire cohort; preoperative anemia was an independent predictor of worse OS and CSS in the US cohort alone, Chinese cohort alone and the combined cohort. We developed a predictive nomogram for OS which exhibited better prognostic value when it included the values of sodium and anemia, and successfully validated it in different cohorts.

Conclusion: Preoperative low serum sodium and anemia could be informative in predicting worse pathologic and survival outcomes in different UTUC patient ethnic groups.

Keywords: upper tract urothelial carcinoma, serum sodium, anemia, survival, China, the United States

\section{Introduction}

Upper tract urothelial carcinoma (UTUC) is a relatively uncommon malignancy, accounting for only $5-10 \%$ of all urothelial carcinomas. ${ }^{1,2}$ Radical nephroureterectomy (RNU) with excision of the bladder cuff is the reference standard procedure for adequate local tumor control and better long-term survival for patients with bulky, high grade or invasive UTUC. ${ }^{3,4}$ Unfortunately, although surgical and medical management has improved, the 5-year cancer-specific mortality rate remains $20-30 \%,{ }^{5,6}$ and $22-47 \%$ of all UTUC patients experience intravesical recurrence (IVR) after RNU, ${ }^{7-9}$ which indicates that selected patients may benefit from early systemic therapy. 
Given the impaired renal function after RNU, neoadjuvant chemotherapy has been recommended in recent years. Traditional prognostic factors, such as pathological tumor stage, grade, lymph node metastasis and lymphovascular invasion (LVI), are inadequate for precise risk stratification and difficult to define before surgery. ${ }^{3}$ This warrants the need for simple and effective preoperative prognostic predictors.

Complete blood tests (CBT) and serum electrolytes are regularly examined prior to RNU in most centers worldwide and might provide potentially useful prognostic blood biomarkers. Studies of UTUC focusing on clinical significance of serum markers including sodium $^{10}$ and serum hemoglobin ${ }^{11-14}$ provide evidence that these two factors might be associated with worse prognosis. ${ }^{15,16}$ However, these studies are limited by sample size, in addition to differences between ethnic groups.

A multi-center database that included patients from China and the United States (U.S.) has been constructed and it is demonstrated that there were significant differences in clinical and pathological characteristics, and predictive models for adverse pathological outcomes. ${ }^{17,18}$ It would also be interesting to illustrate the difference in laboratory values and their prognostic significance between these two cohorts.

In this study, we analyzed the relationship between preoperative serum markers, clinicopathologic characteristics, and prognosis in patients derived from a multi-center international cohort in China and the US.

\section{Patients and Methods}

\section{Patient Enrollment and Evaluation}

After approval by the institutional review board, we retrospectively reviewed the records of 775 consecutive patients diagnosed histologically with UTUC at a single tertiary care medical facility in China $(\mathrm{n}=451,2002-2015)$ or at one of two tertiary care medical facilities in the US $(n=324$, 1998-2015). Patients with incomplete clinical data, synchronous bilateral UTUC or solitary renal units were excluded. No patients received conservative treatment before distal ureterectomy or RNU. All Chinese patients underwent standard RNU with bladder cuff resection. The indications for distal ureterectomy versus RNU in the US were based on surgical judgment and, factors including tumor size, location, likelihood of invasion, multifocality and surgeon experience. Lymphadenectomy, with tumor location dictating boundaries of node dissection, was performed when enlarged lymph nodes were found by preoperative imaging or intraoperative observation. Patient clinicopathological data, including demographics, comorbidities, preoperative imaging characteristics, serum laboratory values (estimated glomerular filtration rate [eGFR], sodium and hemoglobin) and final pathological characteristics were collected. The detailed information about the study samples is available in previous publications from the same cohort. ${ }^{17,18}$

Preoperative anemia was defined as serum hemoglobin $<12 \mathrm{mg} / \mathrm{dL}$ in women and serum hemoglobin $<13 \mathrm{mg} / \mathrm{dL}$ in men based on the World Health Organization classification. The cut-off value for preoperative serum sodium was set at the median value for the overall cohort of patients. ${ }^{19}$ Patients were stratified based on serum hemoglobin level and serum sodium concentration (low serum sodium vs high serum sodium relative to median and non-anemia vs anemia). Staging was assessed according to the 2002 Union for International Cancer Control TNM classification guidelines in China and the American Joint Committee on Cancer for the US cohort, and grade was assessed according to the World Health Organization 1998 consensus classification. The serum laboratory values of all patients were obtained from blood tests within 3 days before surgery. Preoperative renal function (PRF) was measured and recorded as no or mild chronic kidney disease (CKD) $\left(\right.$ GFR $\left.\geq 60 \mathrm{~mL} / \mathrm{min} / 1.73 \mathrm{~m}^{2}\right), \mathrm{CKD}\left(60 \mathrm{~mL} / \mathrm{min} / 1.73 \mathrm{~m}^{2}\right.$ $>\mathrm{eGFR} \geq 15 \mathrm{~mL} / \mathrm{min} / 1.73 \mathrm{~m}^{2}$ ) and end-stage CKD $\left(\right.$ eGFR $\left.<15 \mathrm{~mL} / \mathrm{min} / 1.73 \mathrm{~m}^{2}\right)$.

\section{Follow-Up Protocol}

Follow-up in China was performed every 3 to 6 months for the first 3 years and annually thereafter. Follow-up assessments for recurrence in the US were performed every 3 to 4 months in postoperative year one, semiannually in year two and annually thereafter. Assessments at these time points consisted of history and physical examination, serum chemistry, chest radiography, urinary cytology, cystoscopy and radiographic evaluation of the contralateral upper urinary tract with cross-sectional imaging. Additional imaging tests, including chest computerized tomography, magnetic resonance imaging and bone scans, were obtained as clinically indicated. ${ }^{17}$

Oncologic outcomes, including overall survival (OS), cancer-specific survival (CSS) and intravesical recurrencefree survival (IVRFS), were measured by time-to-event across all time points. And the initial point was defined as the surgery day. The cause of death was measured by 
death certificate, medical chart review or the treating physician. IVR in patients who did not receive prior radical cystectomy (RC) was defined as the detection of a subsequent urothelium carcinoma of the bladder (UCB) upon cystoscopy, with pathological confirmation.

\section{Statistical Analysis}

All statistical tests were performed using SPSS 22.0 (IBM Corp, Armonk, NY, USA) or R i386 2.15.3 (R Foundation for Statistical Computing, http://www.r-project.org) and statistical significance was set at $p<0.05$. Pearson's test and chi-square test were used to determine the distribution of categorical variables, and the Mann-Whitney $U$-test was used for continuous variables. Patients who received chemotherapy were excluded from survival analysis, and patients who underwent prior $\mathrm{RC}$ were excluded from IVRFS analysis. Survival curves of OS, CSS, and IVRFS were plotted using the Kaplan-Meier method and analyzed using the Log rank test. Univariable analysis was assessed using the Log rank test and multivariable analysis was evaluated using the Cox proportional hazard regression model. Nomograms and calibration plots were performed using the "rms" library in R i386 2.15.3, and discrimination was measured using Harrell's concordance index (c-statistic). Only those variables that were identified as $p<0.1$ in the univariable analysis were included in the multivariable analysis.

\section{Result}

\section{Patient Characteristics}

A total of 682 (88.0\%) patients with UTUC were enrolled across all 3 institutions. The patients had complete information regarding preoperative serum sodium concentration and hemoglobin (Table 1). Preoperative serum sodium concentration ranged from 129.0 to $149.4 \mathrm{mEq} / \mathrm{L}$, with a median of $140 \mathrm{mEq} / \mathrm{L}$ in the overall cohort which would be used to define low serum sodium in the following analysis. $44.3 \%$ (302/682) of patients had low preoperative serum sodium, and $46.2 \%(315 / 682)$ had preoperative anemia. The US patients had lower serum sodium concentrations compared with Chinese patients $(58.9 \%$ vs $36.8 \%$, p $<$ $0.001)$. The incidence of anemia was comparable between the two cohorts $(47.9 \%$ vs $45.3 \%, \mathrm{p}=0.519)$.

\section{Associations of Preoperative Low Serum Sodium and Anemia with Clinicopathological Characteristics}

Supplementary Tables 1 and Tables 2 the associations of preoperative serum sodium and hemoglobin with clinicopathological characteristics, respectively. Low serum sodium concentration was assocated with Caucasian ethnicity $(\mathrm{p}<$ $0.001)$, cigarette smoking $(p<0.001)$, greater risk of hypertension $(\mathrm{p}=0.009)$, worse American statistical association (ASA) score $(p=0.017)$, tumor multifocality $(p=0.024)$, presence of LVI $(p=0.045)$ and presence of $\mathrm{N} 1$ (compared to $\mathrm{Nx} / \mathrm{N} 0$, $p=0.004)$ in the entire cohort, and specifically non-Caucasian ethnicity $(p=0.033)$ and worse ASA score $(p=0.031)$ in American patients, as well as better ASA score $(p=0.023)$ and worse PRF ( $p=0.038)$ in Chinese patients.

In addition, anemia was associated with advanced age $(\mathrm{p}<$ $0.001)$, lower body mass index (BMI) $(p=0.011)$, worse ASA score $(p<0.001)$, greater risk of diabetes mellitus $(p=0.013)$ or hypertension ( $p=0.025)$, worse PRF ( $<<0.001)$, tumor multifocality $(p=0.005)$, high tumor grade $(p=0.012)$ and presence of LVI $(p=0.041)$ in the entire cohort. Anemia was associated with non-Caucasian ethnicity $(\mathrm{p}=0.026)$, lower BMI $(\mathrm{p}=0.021)$ worse ASA score $(p=0.001)$, worse PRF $(p<0.001)$ and presence of LVI $(p=0.030)$ in American patients. In Chinese patients, anemia was associated with advanced age $(\mathrm{p}<0.001)$, lower BMI $(p=0.044)$, worse ASA score $(p<0.001)$, worse

Table I Data of Serum Markers Stratified by Country

\begin{tabular}{|c|c|c|c|c|}
\hline Variables & Total & The U.S. & China & p value \\
\hline Total patients, $\mathrm{n}$ & 775 & 324 & 451 & - \\
\hline Serum sodium $* *, n(\%)$ & $682(88.0)$ & 231 (7I.3) & $45 I(100)$ & - \\
\hline$\geq 140 \mathrm{mEq} / \mathrm{L}, \%$ & & $4 I . I(95 / 231)$ & $63.2(285 / 45 I)$ & $<0.00 I^{*}$ \\
\hline$<140 \mathrm{mEq} / \mathrm{L}, \%$ & & $58.9(|36 / 23|)$ & $36.8(|66 / 45|)$ & \\
\hline Serum sodium $* * *, m E q / L$ & $140 \pm 3.1$ & $139.0 \pm 3.0$ & $140.5 \pm 3.0$ & $<0.001 *$ \\
\hline Serum hemoglobin**, n (\%) & $682(88.0)$ & $236(72.8)$ & $446(98.9)$ & - \\
\hline Non-anemia, \% & & $52.1(123 / 236)$ & $54.7(244 / 446)$ & 0.519 \\
\hline Anemia, \% & & $47.9(113 / 236)$ & $45.3(202 / 446)$ & \\
\hline Serum hemoglobin ${ }^{* * *}, g / d L$ & $12.5 \pm 1.9$ & $12.7 \pm 1.8$ & $12.4 \pm 1.9$ & 0.082 \\
\hline
\end{tabular}

Notes: *Statistically significant. **The Pearson's test and the chi-square test were used to determine the distribution of categorical variables. ***The Mann-Whitney U-test was used for continuous variables. 
PRF $(p<0.001)$, greater risk of diabetes mellitus $(p=0.022)$ or hypertension $(p=0.042)$, tumor multifocality $(p=0.026)$ and high tumor grade $(\mathrm{p}=0.015)$.

\section{Survival Analysis}

The median follow-up in China was significantly longer compared with the US, as previously reported, ${ }^{15}$ and US patients more frequently experienced IVR with a shorter duration to relapse and had worse OS. CSS and IVRFS were comparable in both countries. Kaplan-Meier curves revealed that patients with preoperative low serum sodium had better IVRFS ( $p=$ $0.009)$ in the US, but worse OS $(p=0.018)$ in the entire cohort (Figure 1). Univariable Cox analysis found that preoperative low serum sodium was associated with improved IVRFS (HR $0.546,95 \%$ CI $0.343-0.868, \mathrm{p}=0.011$ ) in the US (Table 2), and worse OS (HR 1.417, 95\% CI 1.067-1.833, $\mathrm{p}=0.016)$ in
A

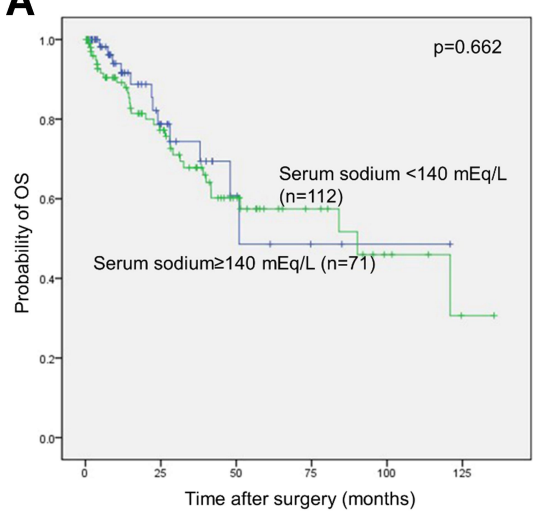

D

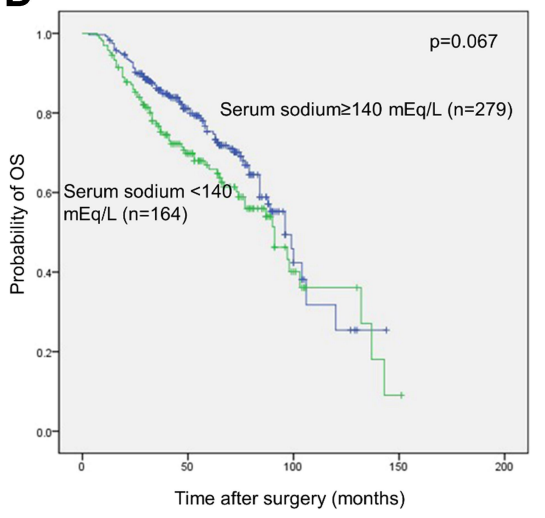

G

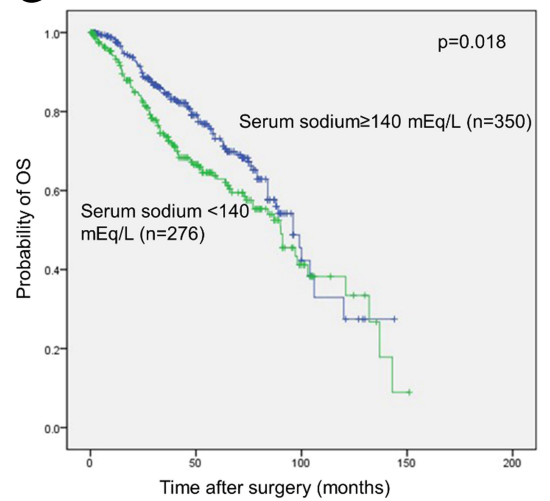

The U.S.

B

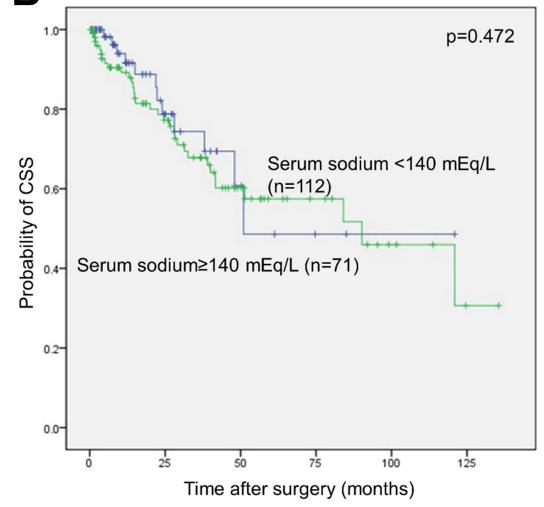

China

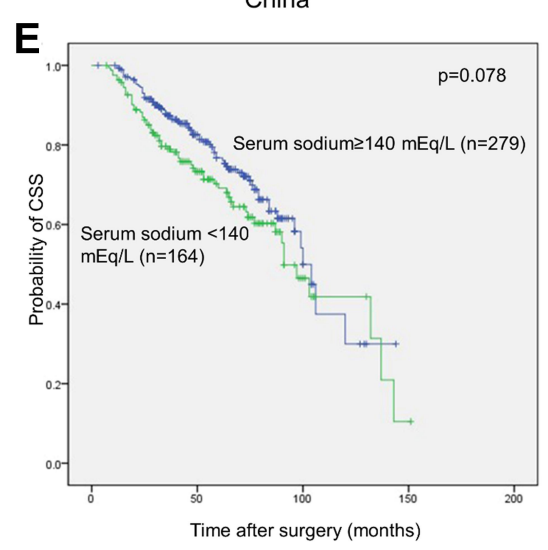

The U.S. and China

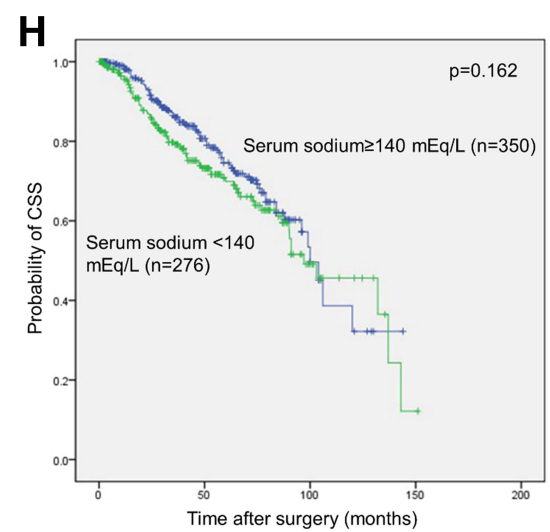

C

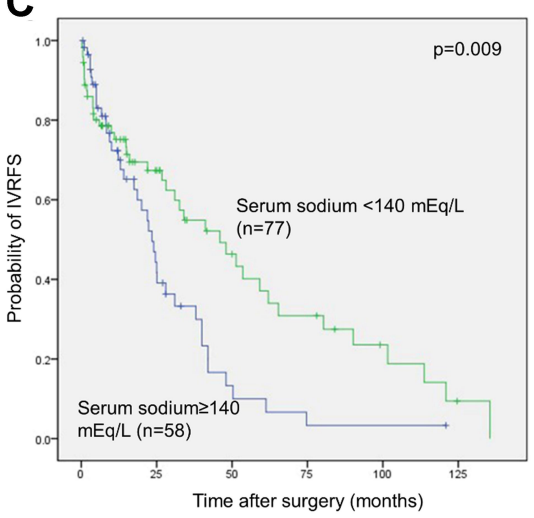

$\mathbf{F}$
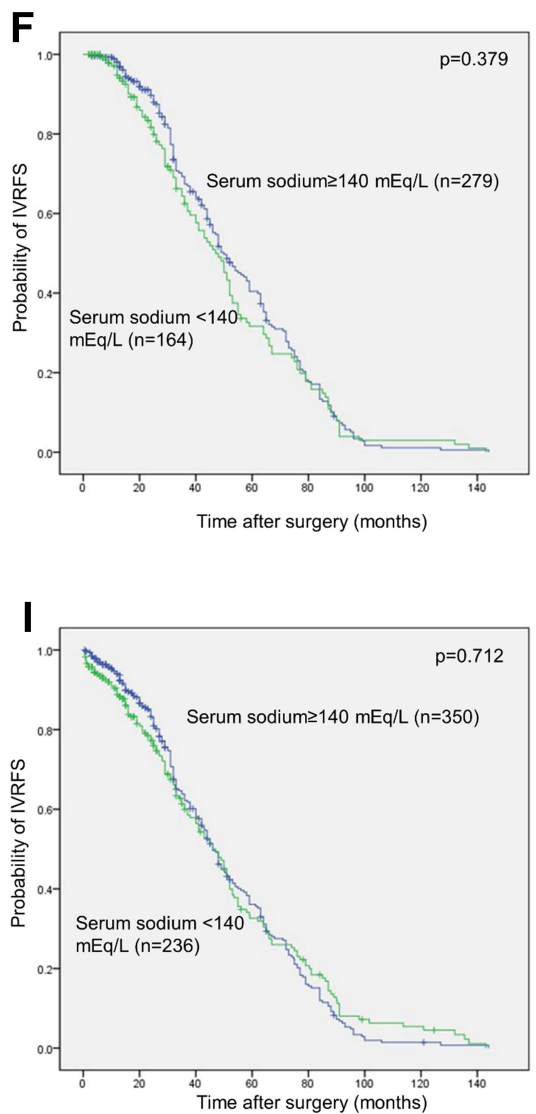

Figure I Oncologic outcomes for patients stratified by preoperative serum sodium level. OS, CSS, and IVRFS are shown for (A-C) the US, (D-F) China, and (G-I) both cohorts together. The dots represent censored observations. 
Table 2 Univariable and Multivariable Analyses of the Correlations Between Preoperative Low Serum Sodium/Anemia and IVRFS in UTUC Patients

\begin{tabular}{|c|c|c|c|c|c|c|}
\hline \multirow[t]{3}{*}{ Variables } & \multicolumn{2}{|l|}{ Entire Patients } & \multicolumn{2}{|l|}{ U.S. } & \multicolumn{2}{|l|}{ China } \\
\hline & $\begin{array}{l}\text { Univariable } \\
\text { Analyses }\end{array}$ & $\begin{array}{l}\text { Multivariable } \\
\text { Analyses }\end{array}$ & $\begin{array}{l}\text { Univariable } \\
\text { Analyses }\end{array}$ & $\begin{array}{l}\text { Multivariable } \\
\text { Analyses }\end{array}$ & $\begin{array}{l}\text { Univariable } \\
\text { Analyses }\end{array}$ & $\begin{array}{l}\text { Multivariable } \\
\text { Analyses }\end{array}$ \\
\hline & HR (95\% Cl), p & HR (95\% Cl), p & HR ( $95 \% \mathrm{Cl}), \mathrm{p}$ & HR (95\% Cl), p & HR (95\% Cl), p & HR (95\% Cl), p \\
\hline Age, $\geq 70$ vs $<70$ & $\begin{array}{l}1.208 \\
(0.998-1.463) \\
0.052\end{array}$ & $\begin{array}{l}1.251 \\
(1.028-1.522) \\
0.025 *\end{array}$ & $\begin{array}{l}1.135 \\
(0.769-1.674) \\
0.524\end{array}$ & & $\begin{array}{l}1.301 \\
(1.037-1.631) \\
0.023^{*}\end{array}$ & $\begin{array}{l}1.279 \\
(1.017-1.607) \\
0.035^{*}\end{array}$ \\
\hline $\begin{array}{l}\text { Gender, Male vs } \\
\text { Female }\end{array}$ & $\begin{array}{l}0.957 \\
(0.789-1.160) \\
0.652\end{array}$ & & $\begin{array}{l}0.930 \\
(0.620-1.393) \\
0.724\end{array}$ & & $\begin{array}{l}0.94 I \\
(0.749-I . \mid 82) \\
0.603\end{array}$ & \\
\hline BMI, $\geq 25.5$ vs $<25.5$ & $\begin{array}{l}0.880 \\
(0.725-1.068) \\
0.194\end{array}$ & & $\begin{array}{l}0.728 \\
(0.459-1.154) \\
0.177\end{array}$ & & $\begin{array}{l}0.864 \\
(0.684-1.091) \\
0.220\end{array}$ & \\
\hline $\begin{array}{l}P R F, \text { eGFR } \geq 60 \text { vs } \\
\text { eGFR }<60\end{array}$ & $\begin{array}{l}\mathrm{I} .030 \\
(0.847-1.252) \\
0.767\end{array}$ & & $\begin{array}{l}0.964 \\
(0.650-1.430) \\
0.855\end{array}$ & & $\begin{array}{l}0.968 \\
(0.768-1.220) \\
0.784\end{array}$ & \\
\hline $\begin{array}{l}\text { Hydronephrosis, Yes } \\
\text { vs No }\end{array}$ & $\begin{array}{l}1.388 \\
(1.142-1.688) \\
0.001 *\end{array}$ & $\begin{array}{l}1.341 \\
(1.099-1.640) \\
0.004 *\end{array}$ & $\begin{array}{l}1.432 \\
(0.943-2.177) \\
0.092\end{array}$ & $\begin{array}{l}1.315 \\
(0.789-2.189) \\
0.293\end{array}$ & $\begin{array}{l}1.402 \\
(1.116-1.761) \\
0.004^{*}\end{array}$ & $\begin{array}{l}1.387 \\
(1.101-1.774) \\
0.005^{*}\end{array}$ \\
\hline $\begin{array}{l}\text { Tumor stage, Ta-I vs } \\
\text { T2-4 }\end{array}$ & $\begin{array}{l}1.139 \\
(0.939-1.381) \\
0.186\end{array}$ & & $\begin{array}{l}1.313 \\
(0.883-1.952) \\
0.178\end{array}$ & & $\begin{array}{l}1.079 \\
(0.853-1.365) \\
0.525\end{array}$ & \\
\hline $\begin{array}{l}\text { Tumor grade, High vs } \\
\text { Low }\end{array}$ & $\begin{array}{l}1.663 \\
(1.160-2.385) \\
0.006 *\end{array}$ & $\begin{array}{l}1.572 \\
(1.075-2.301) \\
0.020 *\end{array}$ & $\begin{array}{l}1.793 \\
(1.122-2.866) \\
0.015^{*}\end{array}$ & $\begin{array}{l}2.205 \\
(1.115-4.359) \\
0.023^{*}\end{array}$ & $\begin{array}{l}1.678 \\
(0.746-3.773) \\
0.211\end{array}$ & \\
\hline $\begin{array}{l}\text { Nodal status, No/Nx } \\
\text { vs NI }\end{array}$ & $\begin{array}{l}1.688 \\
(1.051-2.079) \\
0.030 *\end{array}$ & $\begin{array}{l}1.496 \\
(0.877-2551) \\
0.139\end{array}$ & $\begin{array}{l}1.655 \\
(0.522-5.25 I) \\
0.392\end{array}$ & & $\begin{array}{l}1.623 \\
(0.965-2.731) \\
0.068\end{array}$ & $\begin{array}{l}1.786 \\
(1.059-3.014) \\
0.030 *\end{array}$ \\
\hline LVI, Yes vs No & $\begin{array}{l}1.428 \\
(1.067-1.911) \\
0.016^{*}\end{array}$ & $\begin{array}{l}1.243 \\
(0.908-1.700) \\
0.175\end{array}$ & $\begin{array}{l}2.301 \\
(1.387-3.818) \\
0.001 *\end{array}$ & $\begin{array}{l}1.440 \\
(0.818-2.537) \\
0.206\end{array}$ & $\begin{array}{l}1.040 \\
(0.716-1.510) \\
0.838\end{array}$ & \\
\hline $\begin{array}{l}\text { Multifocality, Yes vs } \\
\text { No }\end{array}$ & $\begin{array}{l}1.009 \\
(0.700-1.455) \\
0.961\end{array}$ & & $\begin{array}{l}1.037 \\
(0.686-1.569) \\
0.862\end{array}$ & & $\begin{array}{l}0.889 \\
(0.672-1.178) \\
0.413\end{array}$ & \\
\hline Anemia, Yes vs No & $\begin{array}{l}0.990 \\
(0.808-1.212) \\
0.920\end{array}$ & & $\begin{array}{l}0.998 \\
(0.627-|.59|) \\
0.994\end{array}$ & & $\begin{array}{l}\text { I. } 109 \\
(0.877-1.402) \\
0.388\end{array}$ & \\
\hline $\begin{array}{l}\text { Serum sodium, } \geq 140 \\
\text { vs }<140\end{array}$ & $\begin{array}{l}1.143 \\
(0.936-1.395) \\
0.186\end{array}$ & & $\begin{array}{l}0.546 \\
(0.343-0.868) \\
0.011^{*}\end{array}$ & $\begin{array}{l}0.474 \\
(0.288-0.781) \\
0.003 *\end{array}$ & $\begin{array}{l}1.150 \\
(0.918-1.440) \\
0.226\end{array}$ & \\
\hline
\end{tabular}

Note: *Statistically significant.

Abbreviations: BMI, body mass index; PRF, preoperative renal function; eGFR, estimated glomerular filtration rate; LVI, lymphovascular invasion.

the entire cohort (Table 3). Multivariable Cox analysis further $1.043-1.868, \mathrm{p}=0.025$ ) in the entire cohort (Table 3), but confirmed that preoperative low serum sodium was an inde- $\quad$ better IVRFS (HR 0.474, 95\% CI 0.288-0.781, p $=0.003$ ) in pendent predictor of worse OS (HR 1.396, 95\% CI the US cohort (Table 2). 
Table 3 Univariable and Multivariable Analyses of the Correlations Between Preoperative Low Serum Sodium/Anemia and OS in UTUC Patients

\begin{tabular}{|c|c|c|c|c|c|c|}
\hline \multirow[t]{3}{*}{ Variables } & \multicolumn{2}{|l|}{ Entire Patients } & \multicolumn{2}{|l|}{ U.S. } & \multicolumn{2}{|l|}{ China } \\
\hline & $\begin{array}{l}\text { Univariable } \\
\text { Analyses }\end{array}$ & $\begin{array}{l}\text { Multivariable } \\
\text { Analyses }\end{array}$ & $\begin{array}{l}\text { Univariable } \\
\text { Analyses }\end{array}$ & $\begin{array}{l}\text { Multivariable } \\
\text { Analyses }\end{array}$ & $\begin{array}{l}\text { Univariable } \\
\text { Analyses }\end{array}$ & $\begin{array}{l}\text { Multivariable } \\
\text { Analyses }\end{array}$ \\
\hline & HR (95\% CI), p & HR (95\% CI), p & HR (95\% CI), p & HR $(95 \%$ CI), p & HR (95\% CI), p & HR (95\% Cl), p \\
\hline Age, $\geq 70$ vs $<70$ & $\begin{array}{l}1.583 \\
(1.212-2.068) \\
0.001 *\end{array}$ & $\begin{array}{l}1.560 \\
(1.155-2.107) \\
0.004 *\end{array}$ & $\begin{array}{l}I .621 \\
(0.988-2.658) \\
0.056\end{array}$ & $\begin{array}{l}2.034 \\
(0.983-4.208) \\
0.056\end{array}$ & $\begin{array}{l}1.581 \\
(1.141-2.190) \\
0.006 *\end{array}$ & $\begin{array}{l}1.470 \\
(1.045-2.068) \\
0.027^{*}\end{array}$ \\
\hline $\begin{array}{l}\text { Gender, Male vs } \\
\text { Female }\end{array}$ & $\begin{array}{l}1.391 \\
(1.063-1.821) \text {, } \\
0.016^{*}\end{array}$ & $\begin{array}{l}1.606 \\
(1.186-2.175) \\
0.002 *\end{array}$ & $\begin{array}{l}0.991 \\
(0.602-1.629), \\
0.970\end{array}$ & & $\begin{array}{l}1.516 \\
(1.097-2.094) \\
0.012 *\end{array}$ & $\begin{array}{l}1.516 \\
(1.080-2.128) \text {, } \\
0.016 *\end{array}$ \\
\hline BMI, $\geq 25.5$ vs $<25.5$ & $\begin{array}{l}1.014 \\
(0.772-1.332) \\
0.922\end{array}$ & & $\begin{array}{l}0.785 \\
(0.447-1.378) \\
0.399\end{array}$ & & $\begin{array}{l}1.008 \\
(0.724-1.403) \\
0.965\end{array}$ & \\
\hline $\begin{array}{l}\text { PRF, eGFR } \geq 60 \text { vs } \\
\text { eGFR }<60\end{array}$ & $\begin{array}{l}1.628 \\
(1.242-2.134) \\
<0.00 I^{*}\end{array}$ & $\begin{array}{l}1.131 \\
(0.822-1.556), \\
0.450\end{array}$ & $\begin{array}{l}1.765 \\
(1.066-2.923) \\
0.027^{*}\end{array}$ & $\begin{array}{l}0.856 \\
(0.407-1.800) \\
0.682\end{array}$ & $\begin{array}{l}1.502 \\
(1.083-2.084) \\
0.015^{*}\end{array}$ & $\begin{array}{l}1.179 \\
(0.815-1.705) \\
0.381\end{array}$ \\
\hline $\begin{array}{l}\text { Hydronephrosis, } \\
\text { Yes vs No }\end{array}$ & $\begin{array}{l}1.633 \\
(1.237-2.157) \\
0.001 *\end{array}$ & $\begin{array}{l}1.553 \\
(1.132-2.130) \\
0.006^{*}\end{array}$ & $\begin{array}{l}2.215 \\
(1.325-3.703) \\
0.002 *\end{array}$ & $\begin{array}{l}2.283 \\
(1.101-4.735) \\
0.026^{*}\end{array}$ & $\begin{array}{l}1.604 \\
(1.145-2.248) \\
0.006 *\end{array}$ & $\begin{array}{l}1.372 \\
(0.956-1.967) \\
0.086\end{array}$ \\
\hline $\begin{array}{l}\text { Tumor stage, Ta-I } \\
\text { vs T2-4 }\end{array}$ & $\begin{array}{l}2.105 \\
(1.572-2.818), \\
<0.00 I^{*}\end{array}$ & $\begin{array}{l}1.959 \\
(1.373-2.794) \\
<0.001 *\end{array}$ & $\begin{array}{l}2.727 \\
(1.692-4.395) \\
<0.001 *\end{array}$ & $\begin{array}{l}1.879 \\
(0.840-4.205) \\
0.125\end{array}$ & $\begin{array}{l}2.254 \\
(1.516-3.350) \\
<0.001 *\end{array}$ & $\begin{array}{l}2.134 \\
(1.417-3.214) \\
<0.001 *\end{array}$ \\
\hline $\begin{array}{l}\text { Tumor grade, High } \\
\text { vs Low }\end{array}$ & $\begin{array}{l}\text { I.634 } \\
(1.004-2.659) \\
0.048^{*}\end{array}$ & $\begin{array}{l}\text { I.534 } \\
(0.702-3.35 I) \\
0.283\end{array}$ & $\begin{array}{l}1.820 \\
(1.022-3.240) \\
0.042 *\end{array}$ & $\begin{array}{l}\text { I.578 } \\
(0.568-4.384) \\
0.38 \mid\end{array}$ & $\begin{array}{l}3.062 \\
(0.756-12.40) \\
0.117\end{array}$ & \\
\hline $\begin{array}{l}\text { Nodal status, } \mathrm{N} 0 / \\
\mathrm{Nx} \text { vs NI }\end{array}$ & $\begin{array}{l}3.274 \\
(1.899-5.643) \\
<0.00 I^{*}\end{array}$ & $\begin{array}{l}2.285 \\
(1.173-4.452) \\
0.015^{*}\end{array}$ & $\begin{array}{l}5.109 \\
(2.177-11.99) \\
<0.001 *\end{array}$ & $\begin{array}{l}2.527 \\
(0.790-8.083), \\
0.118\end{array}$ & $\begin{array}{l}2.045 \\
(1.294-3.409) \\
0.008^{*}\end{array}$ & $\begin{array}{l}2.267 \\
(I .09|-4.7| 2) \\
0.028 *\end{array}$ \\
\hline LVI, Yes vs No & $\begin{array}{l}2.087 \\
(1.445-3.013) \\
<0.00 I^{*}\end{array}$ & $\begin{array}{l}\text { I.148 } \\
(0.719-1.833) \\
0.562\end{array}$ & $\begin{array}{l}3.756 \\
(2.151-6.557) \\
<0.00 I^{*}\end{array}$ & $\begin{array}{l}1.547 \\
(0.688-3.480) \\
0.291\end{array}$ & $\begin{array}{l}1.283 \\
(0.762-2.160) \\
0.348\end{array}$ & \\
\hline $\begin{array}{l}\text { Multifocality, Yes vs } \\
\text { No }\end{array}$ & $\begin{array}{l}1.231 \\
(0.920-1.646) \\
0.161\end{array}$ & & $\begin{array}{l}1.359 \\
(0.822-2.248) \\
0.232\end{array}$ & & $\begin{array}{l}1.106 \\
(0.769-1.591) \\
0.588\end{array}$ & \\
\hline $\begin{array}{l}\text { Serum sodium, } \\
<140 \text { vs } \geq 140\end{array}$ & $\begin{array}{l}1.417 \\
(1.067-1.883) \\
0.016 *\end{array}$ & $\begin{array}{l}1.469 \\
(1.091-1.979) \\
0.011^{*}\end{array}$ & $\begin{array}{l}\text { I. } 275 \\
(0.638-2.547) \\
0.492\end{array}$ & & $\begin{array}{l}1.351 \\
(0.977-1.870) \\
0.069\end{array}$ & $\begin{array}{l}1.321 \\
(0.945-1.847) \\
0.103\end{array}$ \\
\hline Anemia, Yes vs No & $\begin{array}{l}1.886 \\
(1.41 \mathrm{I}-2.522) \\
<0.00 \mathrm{I}^{*}\end{array}$ & $\begin{array}{l}1.791 \\
(1.296-2.477) \\
<0.001 *\end{array}$ & $\begin{array}{l}2.655 \\
(1.425-4.945), \\
0.002 *\end{array}$ & $\begin{array}{l}2.804 \\
(1.42 I-5.534) \\
0.003^{*}\end{array}$ & $\begin{array}{l}1.696 \\
(1.219-2.359) \\
0.002 *\end{array}$ & $\begin{array}{l}1.605 \\
(1.110-2.320) \\
0.012 *\end{array}$ \\
\hline
\end{tabular}

Note: *Statistically significant.

Abbreviations: BMI, body mass index; PRF, preoperative renal function; eGFR, estimated glomerular filtration rate; LVI, lymphovascular invasion.

Kaplan-Meier curves revealed that patients with preoperative anemia had worse OS and CSS (all $p<0.05$ ) in the US cohort, Chinese cohort and the entire cohort
(Figure 2). Univariable Cox analysis revealed that preoperative anemia was related to poor survival outcomes in the US cohort (OS: HR 2.728, 95\% CI 1.470-5.061, 

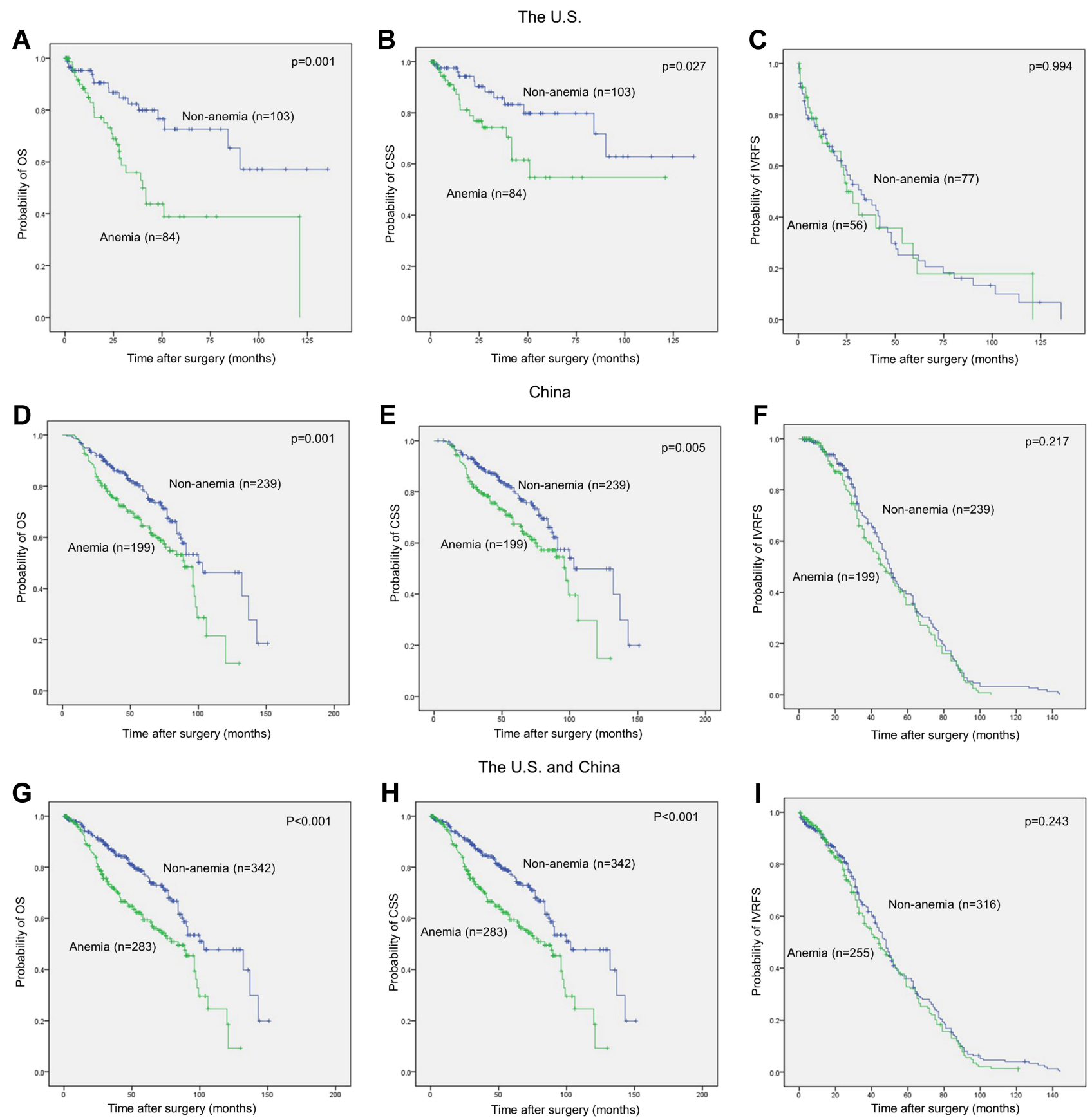

Figure 2 Oncologic outcomes for patients stratified by preoperative anemia. OS, CSS, and IVRFS are shown for (A-C) the US, (D-F) China, and (G-I) both cohorts together. The dots represent censored observations.

$\mathrm{p}=0.001 ;$ CSS: HR 2.256, 95\% CI 1.075-4.737, $\mathrm{p}=0.002)$, Chinese cohort (OS: HR 1.696, 95\% CI $1.219-2.359, \mathrm{p}=0.002$; CSS: HR 1.652, 95\% CI 1.161-2.350, $\mathrm{p}=0.005$ ), and the entire cohort (OS: HR 1.902, 95\% CI 1.423-2.541, p < 0.001; CSS: HR 1.761, 95\% CI 1.282-2.418, p < 0.001) (Tables 3 and 4). Multivariable Cox analysis further revealed that preoperative anemia was an independent predictor of worse
OS and CSS in the US cohort (OS: HR 2.849, 95\% CI 1.448-5.605, $\mathrm{p}=0.002$; CSS: HR 2.493, 95\% CI 1.112-5.589, $\mathrm{p}=0.027$ ), Chinese cohort (OS: HR $1.614,95 \%$ CI 1.119-2.328, $\mathrm{p}=0.010$; CSS: HR $1.646,95 \%$ CI $1.112-2.437, \mathrm{p}=0.013$ ), and the entire cohort (OS: HR 1.761, 95\% CI 1.282-2.418, p < 0.001; CSS: HR 1.744, 95\% CI 1.228-2.478, p $=0.002$ ) (Tables 3 and 4). 
Table 4 Univariable and Multivariable Analyses of the Correlations Between Preoperative Low Serum Sodium/Anemia and CSS in UTUC Patients

\begin{tabular}{|c|c|c|c|c|c|c|}
\hline \multirow[t]{3}{*}{ Variables } & \multicolumn{2}{|l|}{ Entire Patients } & \multicolumn{2}{|l|}{ U.S. } & \multicolumn{2}{|l|}{ China } \\
\hline & $\begin{array}{l}\text { Univariable } \\
\text { Analyses }\end{array}$ & $\begin{array}{l}\text { Multivariable } \\
\text { Analyses }\end{array}$ & $\begin{array}{l}\text { Univariable } \\
\text { Analyses }\end{array}$ & $\begin{array}{l}\text { Multivariable } \\
\text { Analyses }\end{array}$ & $\begin{array}{l}\text { Univariable } \\
\text { Analyses }\end{array}$ & $\begin{array}{l}\text { Multivariable } \\
\text { Analyses }\end{array}$ \\
\hline & HR (95\% CI), p & HR (95\% CI), p & HR (95\% CI), p & HR (95\% CI), p & HR (95\% CI), p & HR (95\% Cl), p \\
\hline Age, $\geq 70$ vs $<70$ & $\begin{array}{l}1.464(1.082-1.980) \\
0.013^{*}\end{array}$ & $\begin{array}{l}1.517 \\
(1.090-2.110) \\
0.013^{*}\end{array}$ & $\begin{array}{l}1.843 \\
(0.946-3.590) \\
0.072\end{array}$ & $\begin{array}{l}3.343 \\
(1.236-9.040) \\
0.017^{*}\end{array}$ & $\begin{array}{l}1.478 \\
(1.043-2.094), \\
0.028 *\end{array}$ & $\begin{array}{l}1.380 \\
(0.958-1.988) \\
0.084\end{array}$ \\
\hline $\begin{array}{l}\text { Gender, Male vs } \\
\text { Female }\end{array}$ & $\begin{array}{l}1.482(1.091-2.013) \\
0.012 *\end{array}$ & $\begin{array}{l}1.807 \\
(1.298-2.515), \\
<0.001 *\end{array}$ & $\begin{array}{l}1.219 \\
(0.617-2.407) \\
0.568\end{array}$ & & $\begin{array}{l}1.642(1.162- \\
2.322), 0.005^{*}\end{array}$ & $\begin{array}{l}1.635 \\
(1.136-2.352), \\
0.008 *\end{array}$ \\
\hline BMI, $\geq 25.5$ vs $<25.5$ & $\begin{array}{l}\mathrm{I} .020(0.75 \mathrm{I}-\mathrm{I} .386) \\
0.897\end{array}$ & & $\begin{array}{l}0.924 \\
(0.433-1.975) \\
0.839\end{array}$ & & $\begin{array}{l}\text { I.II2 } \\
(0.784-1.577), \\
0.552\end{array}$ & \\
\hline $\begin{array}{l}\text { PRF, eGFR } \geq 60 \text { vs } \\
\text { eGFR }<60\end{array}$ & $\begin{array}{l}\text { I.557 (1.147-2.113), } \\
0.005^{*}\end{array}$ & $\begin{array}{l}I .124 \\
(0.794-1.692) \\
0.471\end{array}$ & $\begin{array}{l}2.167 \\
(1.094-4.290) \\
0.027^{*}\end{array}$ & $\begin{array}{l}0.768 \\
(0.297-1.988) \\
0.587\end{array}$ & $\begin{array}{l}1.444 \\
(1.017-2.050), \\
0.040 *\end{array}$ & $\begin{array}{l}1.135 \\
(0.765-1.684), \\
0.529\end{array}$ \\
\hline $\begin{array}{l}\text { Hydronephrosis, } \\
\text { Yes vs No }\end{array}$ & $\begin{array}{l}\text { I.95I (I.422-2.678), } \\
<0.00 I^{*}\end{array}$ & $\begin{array}{l}1.615 \\
(1.135-2.296) \\
0.008 *\end{array}$ & $\begin{array}{l}3.547 \\
(1.829-6.879), \\
<0.00 I^{*}\end{array}$ & $\begin{array}{l}3.375 \\
(1.304-8.736), \\
0.012 *\end{array}$ & $\begin{array}{l}1.642 \\
(1.144-2.356), \\
0.007 *\end{array}$ & $\begin{array}{l}1.420 \\
(0.966-2.088) \\
0.074\end{array}$ \\
\hline $\begin{array}{l}\text { Tumor stage, Ta-l } \\
\text { vs T2-4 }\end{array}$ & $\begin{array}{l}3.062 \\
(2.1134-4.393), \\
<0.001^{*}\end{array}$ & $\begin{array}{l}2.185 \\
(1.460-3.269), \\
<0.001 *\end{array}$ & $\begin{array}{l}5.647 \\
(2.855-11.17), \\
<0.001 *\end{array}$ & $\begin{array}{l}1.974 \\
(0.719-5.419) \\
0.187\end{array}$ & $\begin{array}{l}2.407 \\
(1.565-3.701), \\
0.010 *\end{array}$ & $\begin{array}{l}2.269 \\
(1.454-3.543), \\
<0.001 *\end{array}$ \\
\hline $\begin{array}{l}\text { Tumor grade, High } \\
\text { vs Low }\end{array}$ & $\begin{array}{l}3.915 \text { (1.729-8.863), } \\
0.001 *\end{array}$ & $\begin{array}{l}2.011 \\
(0.728-5.559), \\
0.178\end{array}$ & $\begin{array}{l}4.184 \\
(1.484-11.80), \\
0.007^{*}\end{array}$ & $\begin{array}{l}2.916 \\
(0.617-13.78), \\
0.177\end{array}$ & $\begin{array}{l}2.639 \\
(0.65 I-10.70), \\
0.174\end{array}$ & \\
\hline $\begin{array}{l}\text { Nodal status, N0/ } \\
\text { Nx vs NI }\end{array}$ & $\begin{array}{l}3.614(2.003-6.522) \\
<0.001 *\end{array}$ & $\begin{array}{l}2.064 \\
(0.997-4.276), \\
0.051\end{array}$ & $\begin{array}{l}6.760 \\
(2.605-17.54) \\
<0.00 I^{*}\end{array}$ & $\begin{array}{l}2.393 \\
(0.620-9.234) \\
0.205\end{array}$ & $\begin{array}{l}2.656 \\
(1.236-5.704) \\
0.012^{*}\end{array}$ & $\begin{array}{l}2.211 \\
(1.012-4.831) \\
0.047^{*}\end{array}$ \\
\hline LVI, Yes vs No & $\begin{array}{l}2.295(1.537-3.427), \\
<0.001 *\end{array}$ & $\begin{array}{l}1.217 \\
(0.734-2.016) \\
0.446\end{array}$ & $\begin{array}{l}5.036 \\
(2.581-9.828) \\
<0.00 I^{*}\end{array}$ & $\begin{array}{l}1.528 \\
(0.605-3.862) \\
0.370\end{array}$ & $\begin{array}{l}1.388 \\
(0.808-2.382) \\
0.235\end{array}$ & \\
\hline $\begin{array}{l}\text { Multifocality, Yes vs } \\
\text { No }\end{array}$ & $\begin{array}{l}1.229(0.886-1.705) \\
0.217\end{array}$ & & $\begin{array}{l}\text { I.237 } \\
(0.643-2.379) \\
0.524\end{array}$ & & $\begin{array}{l}\text { I. } 183 \\
(0.807-1.733) \\
0.390\end{array}$ & \\
\hline $\begin{array}{l}\text { Serum sodium, } \\
\geq 140 \text { vs }<140\end{array}$ & $\begin{array}{l}\text { I.262 (0.924-I.724), } \\
0.144\end{array}$ & & $\begin{array}{l}0.822 \\
(0.378-1.787) \\
0.621\end{array}$ & & $\begin{array}{l}1.363 \\
(0.946-1.928) \\
0.080\end{array}$ & $\begin{array}{l}1.320 \\
(0.923-1.889) \\
0.128\end{array}$ \\
\hline Anemia, Yes vs No & $\begin{array}{l}\text { I.742 (I.268-2.394), } \\
0.00 I^{*}\end{array}$ & $\begin{array}{l}1.726 \\
(1.214-2.455) \\
0.002 *\end{array}$ & $\begin{array}{l}2.153 \\
(1.017-4.558) \\
0.045^{*}\end{array}$ & $\begin{array}{l}2.506 \\
(1.107-5.673) \\
0.028^{*}\end{array}$ & $\begin{array}{l}1.652 \\
(1.16 I-2.350) \\
0.005^{*}\end{array}$ & $\begin{array}{l}1.639 \\
(1.104-2.432) \\
0.014^{*}\end{array}$ \\
\hline
\end{tabular}

Note: *Statistically significant.

Abbreviations: BMI, body mass index; PRF, preoperative renal function; eGFR, estimated glomerular filtration rate; LVI, lymphovascular invasion.

We have also tried to re-run the dataset by different method of serum sodium categorizations, such as using $136 \mathrm{mEq} / \mathrm{L}$, or use different medians in two cohorts
(140.9 mEq/L for Chinese patients and $139 \mathrm{mEq} / \mathrm{L}$ for US patients) and found similar results (data not shown). 


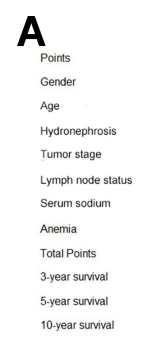

\section{B}

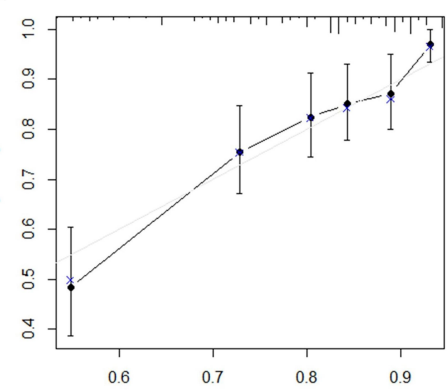

E

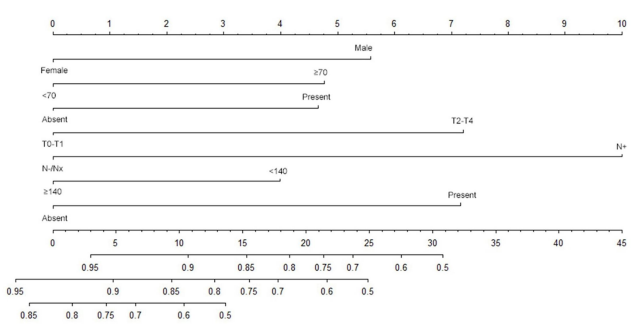

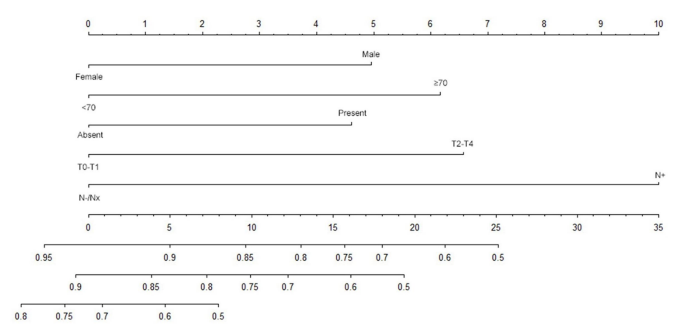

D

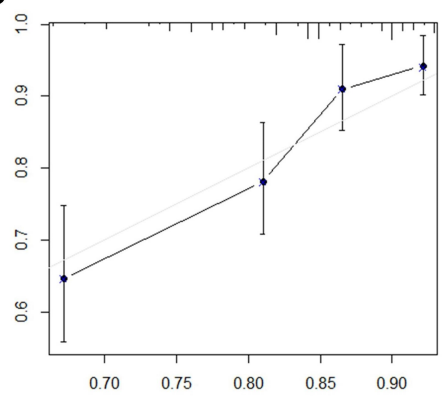

Figure 3 Predictive model for OS: nomogram (A), calibration plot (B), calibration plots in the validation of US cohorts (C) and Chinese cohorts (D), nomogram of the model without information of serum sodium or hemoglobin (E). In nomograms the survival rate was evaluated at 3 years, 5 years and I0 years. In calibration plots survival was evaluated at 3 years.

\section{Risk Classification}

Because preoperative low serum sodium and anemia were independent risk factors for OS in the entire cohort, we constructed a nomogram based on the multivariable analysis. As shown in Figure 3A, the model including laboratory values had a c-statistics of 0.708 . The calibration curve of internal validation was effective (Figure 3B). Validations in the US cohort (Figure 3C) and in the Chinese cohort (Figure 3D) were also performed and the c-statistics were 0.724 and 0.698 , respectively. We also constructed a nomogram that excluded those two factors (Figure 3E) and observed a significantly lower c-statistic of 0.681 .

\section{Discussion}

Based on a large multi-institutional international database of UTUC patients, we analyzed the correlation between preoperative serum markers (sodium and hemoglobin), clinical and pathological characteristics, and prognosis. We found that the US patients had lower baseline serum sodium and comparable hemoglobin compared with Chinese patients. Our results revealed that preoperative low serum sodium was an independent predictor of worse OS in the entire cohort. Preoperative anemia was an independent predictor of worse OS and CSS in the US cohort, Chinese cohort and the entire cohort. Serum sodium and hemoglobin are easily measured and routinely examined, which makes them superior to other preoperative pathologic predictors.

Preoperative low serum sodium - a common tumorrelated electrolyte disturbance-is associated with poor outcomes in cancer patients. ${ }^{20,21}$ Previous studies of renal cell carcinoma demonstrated that preoperative serum sodium levels below the median value were associated with poor OS. ${ }^{22,23}$ In UCB, preoperative serum sodium levels of $\leq 139 \mathrm{mEq} / \mathrm{L}$ were associated with a poor prognosis. ${ }^{19}$ A study of 139 UTUC patients reported that patients with a median serum sodium concentration below $141 \mathrm{mEq} / \mathrm{L}$ had a higher rate of LVI $(\mathrm{p}=0.039)$ and predicted a poor survival. ${ }^{10}$ Meanwhile, previous multicenter studies also indicated that low preoperative serum sodium was associated with poor prognosis. ${ }^{15,16}$ These findings were in accordance with our results. In the present study, the cut-off value of preoperative serum sodium was set at the median value of $140 \mathrm{mEq} / \mathrm{L}$, which was in accordance with previous studies. ${ }^{15,16}$ Preoperative low serum sodium value was related to the presence of LVI and independently predicted a worse OS in the entire cohort.

In the present study, $46.2 \%$ of patients had preoperative anemia, which is consistent with 39.6-65.4\% reported in previous studies. ${ }^{11-14}$ Preoperative anemia was related to tumor multifocality, high tumor grade and presence of LVI. Moreover, it was an independent risk factor for OS 
and CSS in UTUC patients treated with distal ureterectomy or RNU. Our findings validate those in previous studies that preoperative anemia was associated with unfavorable pathological factors and poor oncologic outcomes. ${ }^{11-14,16}$

A low sodium level within the normal range is unlikely to cause poor outcomes in UTUC patients. The mechanisms responsible for decreased serum sodium and the association between preoperative sodium level below the median value and poorer outcomes remain unknown. Chronic inflammation mediated by pro-inflammatory cytokines including interleukin 6 (IL-6) is associated with decreased serum sodium. $^{24}$ In experimental and clinical studies, IL-6 might induce neutrophilia and stimulate the excessive release of antidiuretic hormone (ADH), resulting in decreased serum sodium. $^{22,25}$ In addition, excessive release of $\mathrm{ADH}$ may be mediated by the high tumor burden, which can consequently worsen survival outcomes. $^{26}$

The presence of anemia can aggravate hypoxia in the cancer cell microenvironment, enhance malignant growth and provide a physiological pressure to cancer cells that have lost their apoptotic potential. ${ }^{27}$ In addition, accumulation of HIF-1ainduced by hypoxia is related to poor outcomes in UTUC. ${ }^{28}$ Patients with preoperative anemia have increased plasma vascular endothelial growth factor levels, which may promote angiogenesis in tumors. ${ }^{29}$ Preoperative anemia is associated with aggressive tumor biological features that traditionally serve as poor prognostic factors in UTUC. However, the mechanisms underlying the direct association of preoperative anemia with poorer outcomes remain unclear.

Unlike those relatively unmodifiable prognostic factors, preoperative low serum sodium and anemia may be amenable to correction. Options for sodium supplementation include oral replacement with sodium bicarbonate tablets and vasopressin receptor antagonists. ${ }^{30}$ Options for correcting anemia include transfusions, erythropoiesisstimulating agents and iron supplementations. However, whether the correction of preoperative low serum sodium and anemia could improve survival after surgery remains unknown. Prospective evaluation is warranted.

The present retrospective study would bring information for clinics and works as a fundamental for more multicenter study regarding UTUC in the future. There are some limitations to our study. First, given its retrospective nature, some variables of interest, such as systemic recurrence data, C-reactive protein or aristolochic acid use and dose were not evaluated. Second, as in multi-center analyses, screening guidelines, management patterns and surgical techniques were not standardized and there was a lack of central pathology review, which may have affected histologic interpretation and accuracy of clinical staging between the US and China; however, we have negotiated with the method for laboratory tests and there is a solid foundation for our cooperation about UTUC. Furthermore, the exclusion of patients who received chemotherapy might bring selection bias in two different cohorts. Therefore, the generalizability of our results needs to be validated by larger multi-institutional cohort from the US and China.

\section{Conclusion}

Preoperative low serum sodium and anemia could be informative in predicting worse pathological and survival outcomes in different UTUC patient ethnic groups. This information may help in the selection of patients for receipt of chemotherapy and patient counseling.

\section{Data Sharing Statement}

The datasets of the current study are presented within additional supporting files. Some of them are available from the corresponding author on a reasonable request.

\section{Ethics Approval}

This study was approved by the Ethics Committee of all three center (Peking University First Hospital, University of Texas Southwestern Medical Center and Penn State Milton S. Hershey Medical Center). All procedures performed in studies involving human participants were in accordance with the ethical standards of the institutional research committee and the 1964 Declaration of Helsinki and its later amendments, and written informed consents for review of their medical records were also obtained from all patients.

\section{Acknowledgments}

The authors thank the entire staff of the Department of Urology, Peking University First Hospital, University of Texas Southwestern Medical Center, Beijing Jishuitan Hospital and Penn State Milton S. Hershey Medical Center. We thank Dr Alla Bradley from Liwen Bianji, Edanz Editing China (www.liwenbianji.cn/ac), for editing a draft of this manuscript. 


\section{Funding}

This research did not receive any specific grant from funding agencies in the public, commercial, or not-forprofit sectors.

\section{Disclosure}

The authors declare that they have no conflicting interests for this work.

\section{References}

1. Rouprêt M, Babjuk M, Compérat E, et al. European Association of urology guidelines on upper urinary tract urothelial carcinoma: 2017 update. Eur Urol. 2018;73(1):111-122. doi:10.1016/j.eururo.2017. 07.036

2. Siegel RL, Miller KD, Dvm AJ. Cancer statistics, 2017. CA Cancer J Clin. 2017;67(1):7-30. doi:10.3322/caac.21387

3. Li CC, Chang TH, Wua WJ, et al. Significant predictive factors for prognosis of primary upper urinary tract cancer after radical nephroureterectomy in taiwanese patients. Eur Urol. 2008;54 (5):1127-1135. doi:10.1016/j.eururo.2008.01.054

4. Vitaly M, Shariat SF, Matin SF, et al. Outcomes of radical nephroureterectomy: a series from the upper tract urothelial carcinoma collaboration. Cancer. 2010;115(6):1224-1233. doi:10.1002/cncr. 24135

5. Rouprêt M, Hupertan V, Seisen T, et al. Prediction of cancer specific survival after radical nephroureterectomy for upper tract urothelial carcinoma: development of an optimized postoperative nomogram using decision curve analysis. J Urol. 2013;189(5):1662-1669. doi:10.1016/j.juro.2012.10.057

6. Ploussard G, Xylinas E, Lotan Y, et al. Conditional survival after radical nephroureterectomy for upper tract carcinoma. Eur Urol. 2015;67(4):803-812. doi:10.1016/j.eururo.2014.08.003

7. Ku JH, Choi WS, Kwak C, Kim HH. Bladder cancer after nephroureterectomy in patients with urothelial carcinoma of the upper urinary tract. Urol Oncol. 2011;29(4):383-387. doi:10.1016/j.urolonc.2009. 04.007

8. Xylinas E, Kluth L, Passoni N, et al. Prediction of intravesical recurrence after radical nephroureterectomy: development of a clinical decision-making tool. Eur Urol. 2014;65(3):650-658. doi:10.1016/j.eururo.2013.09.003

9. Xylinas E, Colin P, Audenet F, et al. Intravesical recurrence after radical nephroureterectomy for upper tract urothelial carcinomas: predictors and impact on subsequent oncological outcomes from a national multicenter study. World J Urol. 2013;31(1):61-68. doi:10.1007/s00345-012-0957-3

10. Kazutoshi F, Go T, Ryoichi I, et al. Preoperative serum sodium is associated with cancer-specific survival in patients with upper urinary tract urothelial carcinoma treated by nephroureterectomy. Int J Urol. 2013;20(6):594-601. doi:10.1111/j.1442-2042.2012.03228.x

11. Rink M, Sharifi N, Fritsche HM, et al. Impact of preoperative anemia on oncologic outcomes of upper tract urothelial carcinoma treated with radical nephroureterectomy. J Urol. 2014;191(2):316-322. doi:10.1016/j.juro.2013.09.010

12. Bogomir M, Zoran D, Boris K, Otas D, Uros B, Sandra SG. Prognostic impact of preoperative anemia on urothelial and extraurothelial recurrence in patients with upper tract urothelial carcinoma. Clin Genitourin Cancer. 2015;13(5):485-491. doi:10.1016/j.clgc.2015.03.007

13. Jo JK, Jeong SJ, Hong SK, Byun SS, Lee SE, Oh JJ. The impact of preoperative anemia on oncologic outcome in patients undergoing radical cystectomy for urothelial carcinoma of the bladder. Int Urol Nephrol. 2016;48(4):489-494. doi:10.1007/s11255-016-1219-x
14. Tan P, Xie N, Liao HT, et al. Prognostic impact of preoperative anemia on upper tract urothelial carcinoma. Medicine. 2018;97(37): e12300. doi:10.1097/MD.0000000000012300

15. Fujita K, Uemura M, Yamamoto Y, et al. Preoperative risk stratification for cancer-specific survival of patients with upper urinary tract urothelial carcinoma treated by nephroureterectomy. Int J Clin Oncol. 2015;20(1):156-163. doi:10.1007/s10147-014-0695-1

16. Fujita K, Taneishi $\mathrm{K}$, Inamoto $\mathrm{T}$, et al. Adjuvant chemotherapy improves survival of patients with high-risk upper urinary tract urothelial carcinoma: a propensity score-matched analysis. BMC Urol. 2017;17(1):110. doi:10.1186/s12894-017-0305-4

17. Singla N, Fang D, Su XH, et al. A multi-institutional comparison of clinicopathological characteristics and oncologic outcomes of upper tract urothelial carcinoma in China and the United States. $J$ Urol. 2017;197(5):1208-1213. doi:10.1016/j.juro.2016.11.094

18. Singla N, Fang D, Su XH, et al. Preoperative predictors of nonorgan-confined disease in upper-tract urothelial carcinoma differ between China and the United States. Urol Oncol. 2018;36(3):88. e11-88.e18. doi:10.1016/j.urolonc.2017.10.029

19. Nakata W, Yamamichi G, Tsujimura G, Tsujimoto Y, Nin M, Tsujihata M. Impact of serum sodium concentration on survival outcomes in patients with invasive bladder cancer without metastasis treated by cystectomy. Int J Urol. 2019;26(3):429-431. doi:10.1111/ iju. 13880

20. Doshi SM, Shah P, Lei XD, Lahoti A, Salahudeen AK. Hyponatremia in hospitalized cancer patients and its impact on clinical outcomes. $A m$ J Kidney Dis. 2012;59(2):222-228. doi:10.1053/j.ajkd.2011.08.029

21. Castillo JJ, Glezerman IG, Boklage SH, et al. The occurrence of hyponatremia and its importance as a prognostic factor in a cross-section of cancer patients. BMC Cancer. 2016;16(undefined):564. doi:10.1186/s12885-016-2610-9

22. Kawashima A, Tsujimura A, Takayama H, et al. Impact of hyponatremia on survival of patients with metastatic renal cell carcinoma treated with molecular targeted therapy. Int $J$ Urol. 2012;19 (12):1050-1057. doi:10.1111/j.1442-2042.2012.03115.x

23. Schutz FA, Xie WL, Donskov F, et al. The impact of low serum sodium on treatment outcome of targeted therapy in metastatic renal cell carcinoma: results from the international metastatic renal cell cancer database consortium. Eur Urol. 2014;65(4):723-730. doi:10.1016/j.eururo.2013. 10.013

24. Park SJ, Shin JI. Inflammation and hyponatremia: an underrecognized condition? Korean J Pediatr. 2013;56(12):519-522. doi:10. $3345 / \mathrm{kjp} .2013 .56 .12 .519$

25. Hashizume M, Higuchi Y, Uchiyama Y, Mihara M. IL-6 plays an essential role in neutrophilia under inflammation. Cytokine. 2011;54 (1):92-99. doi:10.1016/j.cyto.2011.01.007

26. Bellmunt J, Leow JJ. Hyponatremia associated with worse outcomes in metastatic renal cell cancer: a potential target for intervention? Eur Urol. 2014;65(4):731-732. doi:10.1016/j.eururo.2013.10.057

27. Graeber TG, Osmanian C, Jacks T, et al. Hypoxia-mediated selection of cells with diminished apoptotic potential in solid tumours. Nature. 1996;379(6560):88-91. doi:10.1038/379088a0

28. Ke HL, Wei YC, Yang SF, et al. Overexpression of hypoxia-inducible factor-1alpha predicts an unfavorable outcome in urothelial carcinoma of the upper urinary tract. Int J Urol. 2008;15(3):200-205. doi:10.1111/j.1442-2042.2007.01978.x

29. Dunst J, Becker A, Lautenschläger C, et al. Anemia and elevated systemic levels of vascular endothelial growth factor (VEGF). Strahlenther Onkol. 2002;178(8):436-441. doi:10.1007/s00066-0020925-8

30. Castillo JJ, Vincent M, Justice E. Diagnosis and management of hyponatremia in cancer patients. Oncologist. 2012;17(6):756-765. doi:10.1634/theoncologist.2011-0400 


\section{Publish your work in this journal}

Cancer Management and Research is an international, peer-reviewed open access journal focusing on cancer research and the optimal use of preventative and integrated treatment interventions to achieve improved outcomes, enhanced survival and quality of life for the cancer patient.

The manuscript management system is completely online and includes a very quick and fair peer-review system, which is all easy to use. Visit http://www.dovepress.com/testimonials.php to read real quotes from published authors.

Submit your manuscript here: https://www.dovepress.com/cancer-management-and-research-journal 